\title{
Dissolved Oxygen Sensing Using an Optical Fiber Long Period Grating Coated With Hemoglobin
}

\author{
Matthew Partridge, Stephen W. James, and Ralph P. Tatam
}

\begin{abstract}
A long period grating fiber optic sensor coated with hemoglobin is used to detect dissolved oxygen. The sensitivity of this sensor to the ratio of dissolved carbon dioxide to dissolved oxygen is demonstrated via the conversion of carboxyhemoglobin to oxyhemoglobin on the sensor surface. The sensor shows good repeatability with a\% $\mathrm{CV}$ of less than $1 \%$ for carboxyhemoglobin and oxyhemoglobin states with no measurable drift or hysteresis.
\end{abstract}

Index Terms-Dissolved gas, dissolved oxygen, fiber optics, haemoglobin, hemoglobin, long period grating, oxygen, sensing.

\section{INTRODUCTION}

D ISSOLVED oxygen sensing is used on a daily basis in a large number of applications in health care and medicine. The most commonly used approach to the measurement of dissolved oxygen in the blood stream is the pulse oximeter, which exploits the absorption of light in the infrared by hemoglobin [1] and typical have a range of $10-90 \%$ oxygenation with a $\pm 5 \%$ error [2]. However, dissolved oxygen measured in this way is only partially useful as a clinical indicator [3]. A measurement of the hemoglobin-available dissolved oxygen would provide a better indication of the actual dissolved oxygen reaching the cells of patients. While rare this is an issue in patients with a range of conditions that cause changes in $\mathrm{pH}$ or temperature of the blood system where normal oxygen uptake is not maintained [4].

Optical fiber based approaches to the sensing of dissolved oxygen include the measurement of changes in the reflectivity of $\mathrm{Ru}(\mathrm{bpy})_{3}$ deposited onto the ends of optical fiber or via tapered evanescent wave sensors [5]. Optical fiber approaches are attractive as a sensor as they can be implanted in specific locations such as cancer growths or specific organs to monitor the blood oxygenation at those sites, which is highly clinically relevant [4].

Long period grating (LPG) have been applied to a wide range of sensing applications from the detection of toluene contamination [6] to the measurement of ammonia gas concentration [7]. They exhibit a high refractive index sensitivity. Without a secondary recognition system, an LPG is inherently nonspecific and will only function as a local refractive index sensor.

Manuscript received December 17, 2015; revised January 26, 2016; accepted February 3, 2016. Date of publication February 22, 2016; date of current version September 22, 2016. This work was supported in part by the Engineering and Physical Sciences Research Council, U.K., under Grant EP/L010437/1. Enquiries for access to the data referred to in this article should be directed to researchdata@cranfield.ac.uk.

The authors are with Engineering Photonics, Cranfield University, Cranfield, MK43 0AL, U.K. (e-mail: m.c.partridge@cranfield.ac.uk; s.w.james@ cranfield.ac.uk; r.p.tatam@cranfield.ac.uk).

Color versions of one or more of the figures in this paper are available online at http://ieeexplore.ieee.org.

Digital Object Identifier 10.1109/JLT.2016.2533161

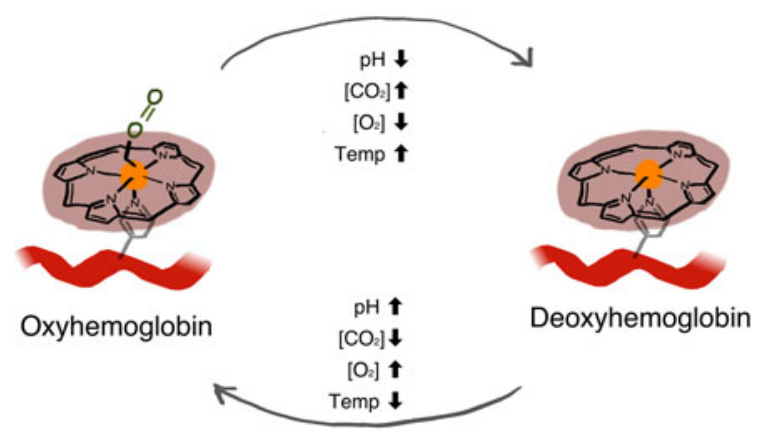

Fig. 1. The conversion of the heme groups in hemoglobin. The influencing factors are listed.

However, when combined with a selective coating they can be sensitized to specific compounds.

Here we examine the use of hemoglobin as the specific sensing coating. Unlike other dissolved oxygen sensitive materials, hemoglobin responds to a number of factors that influence the binding of oxygen (see Fig. 1).

The states pictured in Fig. 1 have a number of competing factors [4] such as $\mathrm{pH}, \mathrm{CO}_{2}$ concentration, $\mathrm{O}_{2}$ concentration and temperature which can drive the hemoglobin from one state to another. These factors are as relevant for hemoglobin in red bloods cells as it is in free solution [8]. Monitoring only one factor such as oxygen concentration only provides a part of the picture about the ability of the blood to take up the oxygen.

Using hemoglobin as the sensing element to detect the uptake of oxygen to cellular hemoglobin is as close a biological homolog as possible, something not possible with simple $\mathrm{Ru}(\mathrm{bpy})_{3}$ based sensors which can only detect the oxygen concentration.

\section{Methods AND Materials}

Preparation of the optical fiber sensor was carried out in two stages. The fabrication of the LPG was followed by the immobilization of hemoglobin onto the surface of the section of the optical fiber containing the LPG.

\section{A. LPG Fabrication}

An LPG was fabricated using a point-by-point irradiation method. A length $(\sim 2 \mathrm{~m})$ of photosensitive fiber (Fiber core PS750) was prepared by stripping the buffer jacket off a short section $(40 \mathrm{~mm})$ in the middle of a $2 \mathrm{~m}$ length. This was then mounted on a translation stage which could be moved with $\sim 0.05 \mu \mathrm{m}$ accuracy. The LPG was inscribed in the fiber core by exposing it to the output from a frequency quadrupled Nd:YAG laser operating at $266 \mathrm{~nm}$. The beam was passed through a slit 


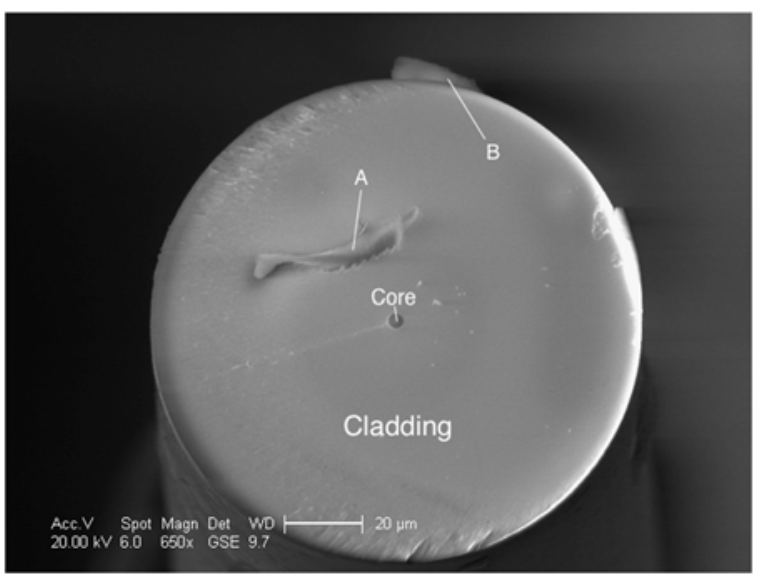

Fig. 2. ESEM picture of the cleaved fiber. A and B highlight portions of the coating material.

of controllable width, manufactured in-house, which was set to a width of $54.85 \mu \mathrm{m}( \pm 0.05)$ which equates to half the desired LPG period of $109.7 \mu \mathrm{m}$ (50\% duty cycle). This period was chosen to ensure the coupling to the LP019 mode near to the phase matching turning point in this fibre type [9]. The fiber was exposed for $40 \mathrm{~s}$ before being translated by a distance equivalent to the desired period.

\section{B. Hemoglobin Coatings}

The hemoglobin used in this experiment was a lyophilized prep from Sigma (H7379) made up to $5 \mathrm{mg} / \mathrm{ml}$ in phosphate buffered saline (PBS) solution. Prior to coating, the fiber was pre-treated with a $0.18 \mathrm{M} \mathrm{KOH}$ solution made up in $60 \%(\mathrm{v} / \mathrm{v})$ ethanol (details) and distilled water. This $\mathrm{KOH}$ treatment is known as a hydroxide etch and ensures that the silica has an abundance of $-\mathrm{OH}$ groups on the exterior surface for protein immobilization. The use of hydroxyl groups to promoted protein absorbance has been well typified [10] and used in ELISA assay design [11].

The treated fiber was then rinsed in distilled water and dried before being immersed in the $5 \mathrm{mg} / \mathrm{ml}$ solution of Hemoglobin. The sensor was then incubated at room temperature $\left(22.5^{\circ} \mathrm{C}\right)$ for $12 \mathrm{~h}$ in sealed container to limit evaporation. After $12 \mathrm{~h}$ the sensor was removed and stored in PBS until use.

Once all the sensor testing had been completed, the fiber was cleaved in the middle of the sensing region and the coating was examined by an environmental scanning electron microscope (ESEM). An image of the coating is shown in Fig. 2.

As can be seen at point $\mathbf{B}$, there is a thin coating layer surrounding the fiber, a flake of the coating disturbed by cleaving the fiber is also identified as $\mathbf{A}$. The size of these features $(\sim 1 \mu \mathrm{m})$ suggest that more than one layer of hemoglobin $(\sim 100 \mathrm{~nm})$ was deposited.

Unlike other protein based selective coatings, such as antibodies, hemoglobin doesn't have external active binding groups. This means that hemoglobin can be deposited in any orientation via passive adherence. Oxygen and carbon dioxide will permeate the protein and reach their binding sites.

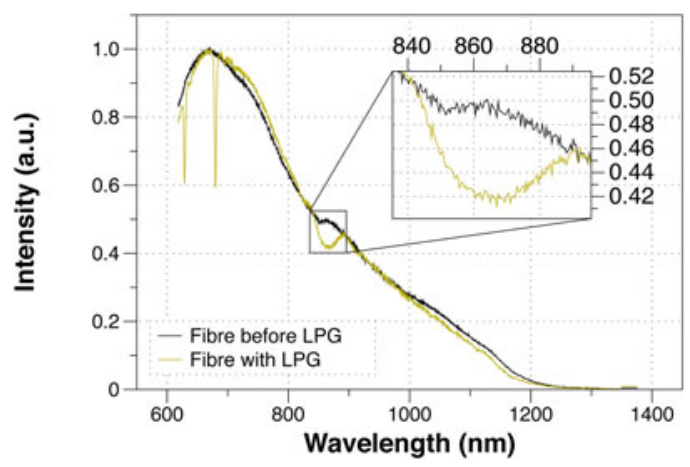

Fig. 3. Spectra recorded before and after LPG fabrication.

\section{Sensor Testing}

For testing, the sensor was mounted in a 3-D printed trough with a volume of $\sim 3 \mathrm{ml}$. The trough was constructed in-house so as to allow the fiber to be held securely either side of the sensing region with the entire sensing region suspended in the center of the container. One end of the fiber was connected to an Ocean Optics tungsten-halogen light source and the other to an Ocean Optics $\mathrm{H} 4000$ spectrophotometer with a range of 600-1100 nm. Each spectrum recorded was an average of the ten scans.

All experiments were carried out in PBS solution (Sigma PBS tablets) dissolved in deionized water. Deionized water will rapidly absorb $\mathrm{CO}_{2}$ from the air after it is produced. To avoid any variation in the dissolved gas content of the water, a single bulk PBS solution was made up which was then 'aerated' by bubbling it with zero-air (BOC) for $3 \mathrm{~h}$.

Previous work by Zhernovaya et al. [12] to typify the refractive index change of hemoglobin used sodium bicarbonate to ensure that all solution-state hemoglobin was converted to oxyhemoglobin, by converting the dissolved carbon dioxide into carbonate groups [13]. 7.5 and $12 \mathrm{mg} / \mathrm{ml}$ solutions of sodium bicarbonate were prepared in aerated PBS.

While the sodium bicarbonate acts on the $\mathrm{CO}_{2}$ converting it to carbonate ions, it has no effect on the dissolved $\mathrm{O}_{2}$. Thus the dissolved oxygen concentration of the solution remains constant throughout the experiment.

\section{RESUltS AND DisCUSSION}

\section{A. LPG Fabrication}

The spectrum of the optical fiber was recorded before and after LPG inscription. The graph shown in Fig. 3 shows these spectra overlaid. The LPG fabrication process took around $3 \mathrm{~h}$, to eliminate any drift in the output from the light source the data was normalized to the maximum value.

The data in Fig. 3 shows the formation of the LP019 phase matching turning point appearing around $865 \mathrm{~nm}$. LP018 and LP017 can be seen appearing at 700 and $610 \mathrm{~nm}$ respectively. LP019 is the most sensitive to local refractive index changes and so only that portion of the spectra is used for subsequent experiments. 


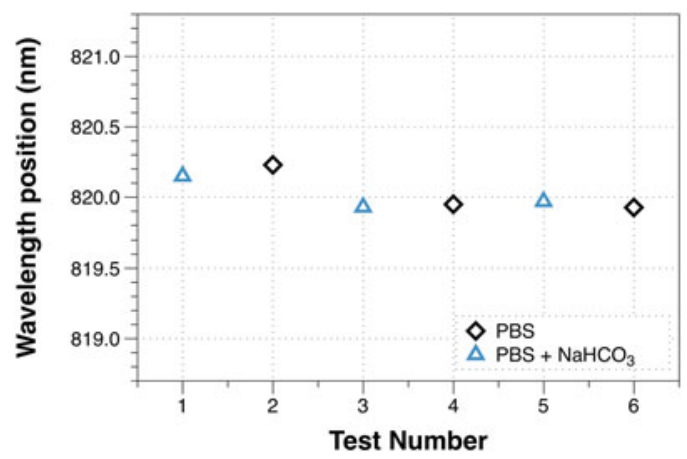

Fig. 4. Wavelength position of the feature around $820 \mathrm{~nm}$ against the test number for PBS and Bicarb solutions.

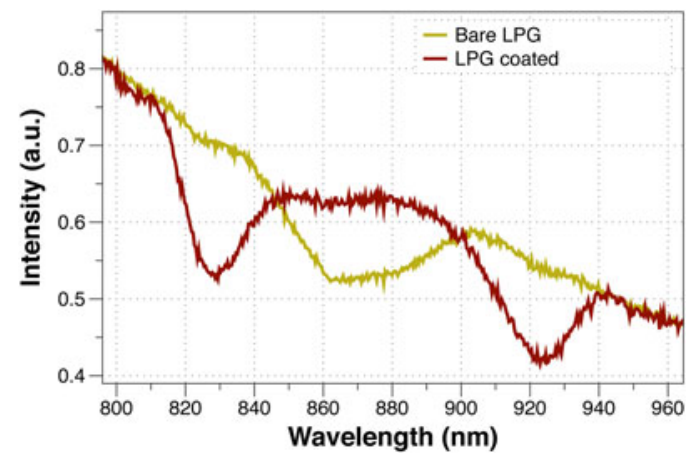

Fig. 5. Transmission spectra of the LPG recorded before and after coating.

\section{B. Non-Specific Reaction Testing}

As previously discussed, LPGs are highly sensitive to refractive index change. To rule out any interference from the bulk refractive index being mistaken for selective sensing, comparator data was taken before coating the sensor.

Prior to $\mathrm{KOH}$ treatment and coating, the LPG was immersed in alternating solutions of PBS and $7.5 \mathrm{mg} / \mathrm{ml}$ sodium bicarbonate solution five times. The position of the peak around $820 \mathrm{~nm}$ was recorded and is plotted in Fig. 4. The peak position was determined by custom data analysis software based on minima location.

There is no discernable difference between the spectra of PBS solution and $7.5 \mathrm{mg} / \mathrm{ml}$ solution, Fig. 4. Without a coating, the LPG cannot differentiate between these solutions.

\section{Hemoglobin Coating}

Fig. 5 shows the normalized transmission spectrum of the LPG recorded before and after coating with hemoglobin. The protein coating causes a local refractive index change significant enough to cause the spectra to shift away from the phasematching turning point (PMTP) to where the coupled modes are shown as separate peaks.

\section{Bicarbonate Testing}

In order to demonstrate that the coated sensor can distinguish between carboxy and oxyhemoglobin, the sensor was immersed in alternating solutions of PBS and $7.5 \mathrm{mg} / \mathrm{ml}$ sodium

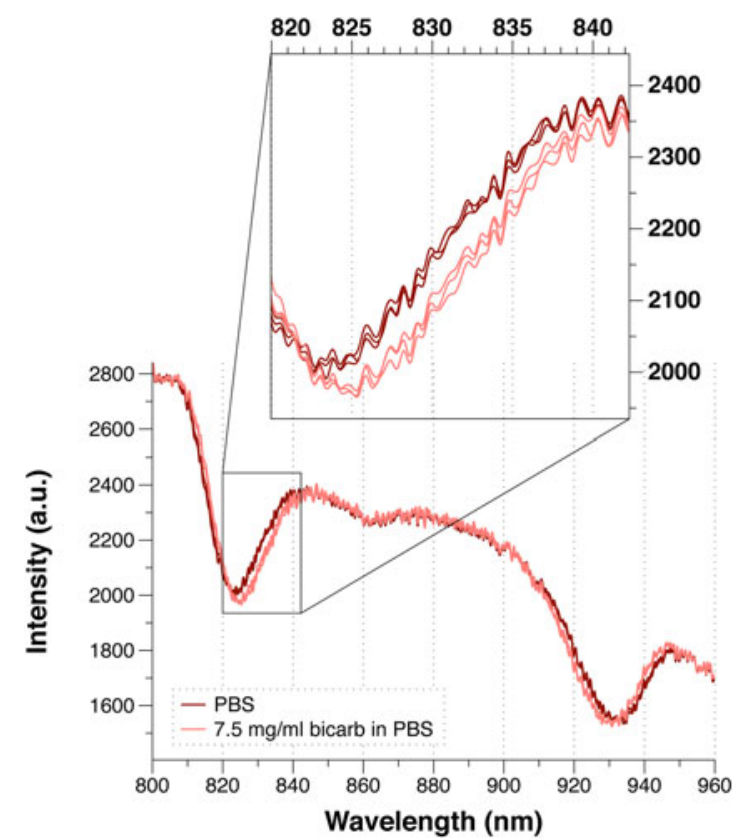

Fig. 6. Transmission spectra of the sensor in PBS and in $7.5 \mathrm{mg} / \mathrm{ml}$ bicarb solution.

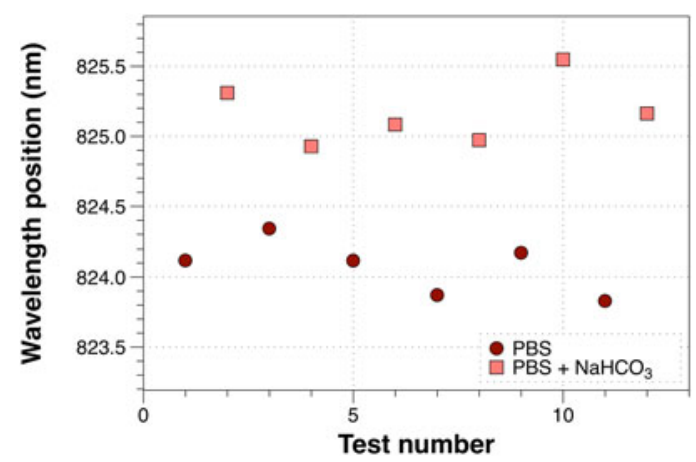

Fig. 7. Wavelength position of the feature around $820 \mathrm{~nm}$ in PBS and $7.5 \mathrm{mg} / \mathrm{ml}$ bicarbonate solution.

bicarbonate solution $(n=6)$. The addition of the sodium bicarbonate should have the effect of shifting the balance from preferential carboxy hemoglobin to oxyhemoglobin [3]. The spectra are plotted in Fig. 6 and the position of the peak around $820 \mathrm{~nm}$ is shown in Fig. 7.

The movement of the coupled LP019 peaks inwards towards the PMTP is indicative of a decrease in the refractive index of the coating [14]. This is in keeping with the decrease in the refractive index of hemoglobin when it converts to oxyhemoglobin [15].

Fig. 7 further demonstrates the discrimination between solutions both of which shows two separate populations of results $(P \leq 0.01)$. By cycling the sensor between the two solutions these results also demonstrate that the sensor is reversible. The standard deviation of $0.3 \mathrm{~nm}$ for PBS and $0.2 \mathrm{~nm}$ for PBS + $\mathrm{NaHCO}_{3}$. There is no trend in either set of results. There is a modulation in the data but this is present in both sets and is possibly due to other conditions affecting the testing. This highlights the need when using LPG sensors to have a secondary control with a non-reactive coating in order to correct for any 


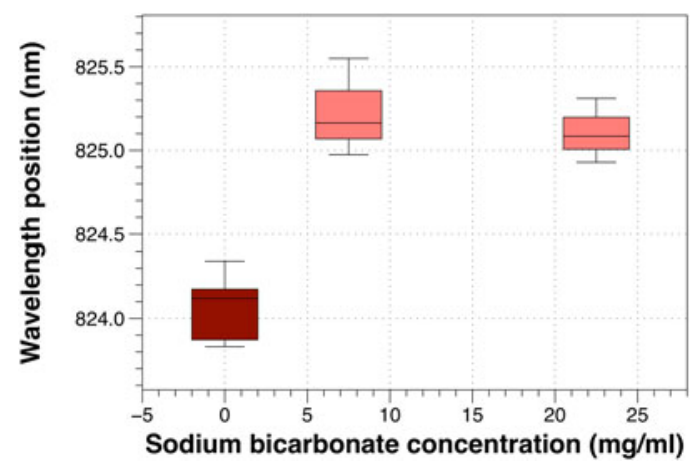

Fig. 8. Wavelength position Box and whisker plot with varying sodium bicarbonate concentration.

possible experimental variation. Other solutions to this include using cascaded LPGs [16].

One possible alternative mechanism for the results produced in Figs. 6 and 7 are that the hemoglobin is not being converted but altered by the inclusion of sodium bicarbonate beyond simple refractive index change. For example, the sodium bicarbonate may have a denaturing effect on the protein causing a conformational, and possibly RI, change without any oxygen absorbance. The $7.5 \mathrm{mg} / \mathrm{ml}$ concentration of sodium bicarbonate was chosen to ensure an excess of sodium bicarbonate to remove the dissolved $\mathrm{CO}_{2}$. A final experiment was carried out using a higher concentration of $22.5 \mathrm{mg} / \mathrm{ml}$ sodium bicarbonate $(n=3)$. The data is shown in Fig. 8.

As expected Fig. 8 shows no change in wavelength position with increasing sodium bicarbonate concentration. This demonstrates that $7.5 \mathrm{mg} / \mathrm{ml}$ is sufficient to maximize the change in hemoglobin and that the results seen are not a result of another effect of the sodium bicarbonate.

\section{CONCLUSION}

A hemoglobin coated LPG sensor for dissolved oxygen has been demonstrated. Initial data shows the sensor's insensitivity to sodium bicarbonate and the deposition of hemoglobin. Further testing with sodium bicarbonate concentrations showed a reaction indicative of the conversion of carboxyhemoglobin to oxyhemoglobin with a shift in the refractive index. A number of possible non-specific chemistries were also ruled out.

The experiments were designed such that the dissolved oxygen content was constant for all solutions. The only changing factor was the dissolved carbon dioxide in solution. This mimic's the same pressures on hemoglobin in the blood stream and show that the hemoglobin deposited on the LPG responded as expected.

This is the first time that hemoglobin has been used as a direct sensing element in conjunction with an optical fiber. Is has been previously applied to electrochemical studies [17] but as a hemoglobin characterization method and not as a sensing element. Future work will examine the response of hemoglobin to other parameters such as $\mathrm{pH}$ and temperature. Also using sodium dithionite [18] it is possible to completely remove dissolved oxygen from samples and demonstrate fully $\mathrm{CO}_{2}$ saturated hemoglobin.
The final goal of this work is to produce an implantable sensor system that can produce real-time feedback on the availability of dissolved oxygen in the blood stream.

\section{REFERENCES}

[1] C. R. Stephen, H. M. Slater, A. L. Johnson, and P. D. Skeklj, "The oximeter-A technical aid for the anesthesiologist," Anesthesiology, vol. 12 , no. 5, pp. 541-555, Sep. 1951.

[2] J. E. Sinex, "Pulse oximetry: Principles and limitations," Amer. J. Emergency Med., vol. 17, pp. 59-67, 1999.

[3] R. N. Pittman, Regulation of Tissue Oxygenation. San Rafael, CA, USA: Morgan \& Claypool, 2011.

[4] J. A. Gutierrez and A. A. Theodorou, "Oxygen delivery and oxygen consumption in pediatric critical care," in Pediatric Critical Care Study Guide: Text and Review. London, U.K.: Springer, 2012.

[5] Y. Xiong, J. Xu, J.-W. Wang, and Y.-F. Guan, "A fiber-optic evanescent wave sensor for dissolved oxygen detection based on novel hybrid fluorinated xerogels immobilized with $[\mathrm{Ru}(\mathrm{bpy}) 3] 2+$," Anal. Bioanal. Chem. vol. 394, no. 3, pp. 919-923, Mar. 2009.

[6] M. Partridge, R. Y. N. Wong, S. W. James, F. Davis, S. P. J. Higson, and R. P. Tatam, "Sensors and Actuators B: Chemical," Sens. Actuators, B. Chem., vol. 203, pp. 621-625, Nov. 2014.

[7] S. O. Korposh and S.-W. Lee, "Fabrication of sensitive fibre-optic gas sensors based on nano-assembled thin films," in Optical Fibre, New Developments, C. Lethien, Ed. Rijeka, Croatia: InTech, 2009.

[8] K. Dalziel and J. R. O'Brien, "The kinetics of deoxygenation of human haemoglobin.," Biochem. J., vol. 78, pp. 236-245, Feb. 1961.

[9] R. Y. N. Wong, E. Chehura, S. E. Staines, S. W. James, and R. P. Tatam, "Fabrication of fiber optic long period gratings operating at the phase matching turning point using an ultraviolet laser," Appl. Opt., vol. 53, no. 21, pp. 4669-4674, 2014.

[10] A. S. Curtis, J. V. Forrester, and C. McInnes, "Adhesion of cells to polystyrene surfaces," J. Cell Biol., vol. 97, pp. 1500-1506, 1983.

[11] H. S. Mansur, R. L. Oréfice, and W. L. Vasconcelos, "Biomaterial with chemically engineered surface for protein immobilization," J. Mater. Sci. Mater. Med., vol. 16, pp. 333-340, 2005.

[12] O. Zhernovaya, O. Sydoruk, V. Tuchin, and A. Douplik, "The refractive index of human hemoglobin in the visible range," Phys. Med. Biol., vol. 56, no. 13, pp. 4013-4021, Jun. 2011.

[13] A. Cents, D. Brilman, and G. F. Versteeg, " $\mathrm{CO}_{2}$ absorption in carbonate/bicarbonate solutions: The Danckwerts-criterion revisited," Chem. Eng. Sci., vol. 60, pp. 5830-5835, 2005.

[14] C. S. Cheung, S. M. Topliss, S. W. James, and R. P. Tatam, "Response of fiber-optic long-period gratings operating near the phase-matching turning point to the deposition of nanostructured coatings," J. Opt. Soc. Amer. B, vol. 25 , no. 6, pp. 897-902, 2008.

[15] O. Sydoruk, O. Zhernovaya, V. Tuchin, and A. Douplik, "Refractive index of solutions of human hemoglobin from the near-infrared to the ultraviolet range: Kramers-Kronig analysis," J. Biomed. Opt, vol. 17, no. 11, p. 115002 , Nov. 2012.

[16] S. W. James, S. O. Korposh, S.-W. Lee, and R. P. Tatam, "A long period grating-based chemical sensor insensitive to the influence of interfering parameters," Opt. Exp., vol. 22, no. 7, pp. 8012-8023, 2014.

[17] H. Sun and N. Hu, "Voltammetric studies of hemoglobin-coated polystyrene latex bead films on pyrolytic graphite electrodes," Biophys. Chem., vol. 110, no. 3, pp. 297-308, Aug. 2004.

[18] C. M. Bright and D. Ellis, "Intracellular $\mathrm{pH}$ changes induced by hypoxia and anoxia in isolated sheep heart Purkinje fibres," Exp. Physiol., vol. 77, no. 1, pp. 165-175, Jan. 1992.

Matthew Partridge received the Ph.D. degree from Cranfield University, Cranfield, U.K., in a joint project between Engineering Photonics and Cranfield Health. He is currently a Research Fellow with the Engineering Photonics Centre, Cranfield University. He has worked extensively in sensor development, including immunoassays, micro arrays, microfluidics, and optical sensors. His current research area is primarily focused on combining novel photonic techniques with Langmuir monolayers to develop improved sensor systems. In addition, he also runs an open science blog and webcomic (errantscience.com) aimed at disseminating complex science to a wider audience. 
Stephen W. James was born in Llanelli, U.K. He received the M.Sc. degree in Applied Optics from Imperial College London, London, U.K., in 1988, and the Ph.D. degree from the University of Southampton, Southampton, U.K., in 1992. He joined Cranfield University, Cranfield, U.K., as a Research Fellow in 1993 , where he developed his interest in the development and application of optical instrumentation and sensors. As a Professor of applied optics, he leads the optical fibre sensing activity within Engineering Photonics Centre, with a focus on sensing chemical and physical parameters and on deploying instrumentation in real-world environments, ranging from foundation piles, railways, and aerospace structures to superconducting magnets.
Ralph P. Tatam received the B.Sc. (Hons.) degree in physics and chemistry from the University of Exeter, Exeter, U.K., and the Ph.D. degree in physics for work on dynamic Rayleigh-Brillouin laser light scattering studies of liquids. He was appointed to a personal Chair in Engineering Photonics Centre, Cranfield University, Cranfield, U.K., in 1998. He worked as a Research Fellow and Lecturer in the Physics Laboratory, University of Kent, developing optical fiber sensors from 1985 to 1989. In 1989, he took up an appointment at Cranfield University, where he has established an internationally recognized research team investigating optical instrumentation and sensors applied to challenging measurement problems in engineering and more recently healthcare. He has published more than 400 publications in journals and conferences. In 2004, he was awarded a D.Sc. by the University of Exeter for his research contributions. $\mathrm{He}$ is a Chartered Engineer, a Fellow of the Institute of Physics, a Fellow of SPIE, and served as an elected member of the Board of Directors of SPIE (2010-2012) 


\section{Dissolved oxygen sensing using an optical fibre long period grating coated with hemoglobin}

\section{Partridge, Matthew}

Institute of Electrical and Electronics Engineers

Matthew Partridge, Stephen James and Ralph Tatam. Dissolved oxygen sensing using an optical fibre long period grating coated with hemoglobin. Journal of Lightwave Technology, Vol.

34, NO. 19, October 2016, pp4506-4510

http://dx.doi.org/10.1109/JLT.2016.2533161

Downloaded from Cranfield Library Services E-Repository 